\title{
Non-Markovian decoherence: A critique of the two-level approximation
}

\author{
T. Hakioğlu ${ }^{\mathrm{a}, *}$, Kerim Savran ${ }^{\mathrm{a}}$, Haldun Sevinçli ${ }^{\mathrm{a}}$, Emine Meşe $^{\mathrm{b}}$ \\ ${ }^{a}$ Department of Physics, Bilkent University, Ankara 06800, Turkey \\ ${ }^{\mathrm{b}}$ Department of Physics, Dicle University, Diyarbakur, Turkey
}

Available online 19 December 2005

\begin{abstract}
The environmental decoherence in multilevelled systems in the context of two-level approximation is examined. It is found that the environmental temperature plays a minor role in the magnitudes of the decoherence rates whereas, the system-environment coupling and the environmental energy spectrum are dominant. Particularly, the latter is important in zero temperature quantum fluctuations and/or the nonequilibrium noise sources due to the large range of energies present in the environmental modes. Decoherence is found to be dominated by the short time nonresonant processes and this observation severely questions the use of the two-levelled models on decoherence.
\end{abstract}

(C) 2005 Elsevier B.V. All rights reserved.

PACS: 03.65.X; 85.25.C; 85.25.D

Keywords: Decoherence; Dephasing; Two-level approximation

\section{Introduction}

Two levelled models are routinely used in the recent applications concerning the decoherence of open quantum systems. The well studied spin-boson [1-4] or central spin $[5,6]$ models are such examples. The general assumption is that for sufficiently low temperatures $T \ll \Delta E$, a multilevelled quantum system (MLS) is well approximated by its first two levels [1] (where $\Delta E$ is a characteristic energy scale separating the first two levels from the higher excited states of the quantum system). On the other hand, the nature of the environmental coupling and the properties of the environmental spectrum are also essential factors in the determination of the decoherence properties in MLS. For realistic MLS under weak environmental perturbation, it is sufficient to consider a Caldeira-Leggett type linear coupling [7-9] of a system coordinate to an environmental coordinate. The spectral range of the environment usually extends to energies well beyond the energy scales of the MLS. Decoherence properties of the MLS is expected to be affected at short times by the entire range of the

\footnotetext{
${ }^{*}$ Corresponding author.

E-mail address: hakioglu@fen.bilkent.edu.tr (T. Hakioğlu).
}

environmental spectrum. This basic short-time property of the system-environment coupling has been largely ignored in the works on decoherence until recently, due to the fact that the environmental correlations have been usually treated as Markovian. A few theoretical approaches now exist on the effect of multilevels in the long time decoherence properties $[10,11]$ ignoring largely the short time behaviour. This long time resonant transition based approach has now become a tradition and the usual practice is to use the Fermi-Golden rule. The calculated decoherence rates, in large, reveal the Markovian subcharacter yielding exponential time behaviour for the reduced density matrix (RDM). The latter is usually solved by analytical methods such as Bloch-Redfield $[12,13]$ or Lindblad [14] operator formalisms, by which the master equation is transformed into a linearly coupled first order ordinary differential equations. Then the corresponding Bloch-Redfield coefficients or the Lindblad operators are time independent with exponential decoherence rates mainly influenced by the resonant sector in the systemenvironment coupling. These analytical methods are easily applicable to systems with large number of levels. On the other hand, the Markov approximation is expected to break down at short observational times. Here short time 
implies times shorter than the typical environmental correlation time below which the system environment coupling is allowed to remember the history of the nonMarkovian time evolution.

In Section 2, we revisit the two-level approximation and recall its basic assumptions for reference. In Section 3, we introduce the multilevel system-environment model. In Section 4, the short time decoherence is investigated. Leakage as a typical multilevel effect is investigated in Section 5 .

\section{2LA revisited}

The 2LA assumes, (a) that the spectrum must have nonnegligible couplings at the right transition frequencies at which the system makes transitions to higher levels; (b) there are no available environmental states to couple with the system at zero or sufficiently low temperatures: this assumption and the notion of right transition frequency enables one to neglect all parts of the spectrum $\Delta E \leqslant \omega$ because of the belief that at sufficiently low temperatures the interacting part of the spectrum is in the low energies $\omega \leqslant T \ll \Delta E$ of which coupling is believed to be suppressed by the much lower temperature; (c) negligible leakage of the wave-function to higher levels: counter arguments against neglecting the leakage effects in the long time dynamics can be found for instance in the recent publications Refs. [11,15]. A number of experiments have also been performed emphasizing the multilevelled nature of those systems particularly used in the context of quantum computation. For instance, current-biased Josephson junctions or rf-SQUID's have been thoroughly investigated in Refs. [16-18]. Similar zero temperature decoherence mechanisms have been recently verified for the mesoscopic persistent current rings experimentally [19] and studied theoretically [20]. In particular, the saturation observed in the electron dephasing rates in disordered conductors [21] has been argued in favour of the zero temperature nonresonant quantum fluctuations [22,23].

\section{A multilevel system-environment model}

The total model Hamiltonian, in the eigenbasis of the system can be written as [10]

$$
\begin{aligned}
H= & \sum_{n=1}^{N} E_{n}|n\rangle\langle n|+\int \mathrm{d} \omega \omega\left(a_{\omega}^{\dagger} a_{\omega}+a_{\omega} a_{\omega}^{\dagger}\right) \\
& +\sum_{n, r=1}^{N} \varphi_{n r}|n\rangle\langle r| \int \mathrm{d} \omega \eta_{\omega}\left(a_{\omega}+a_{\omega}^{\dagger}\right) .
\end{aligned}
$$

Here $E_{n}=n-0.5$ for $1<n$ are fixed system harmonic-like eigenenergies in some absolute (and irrelevant) energy scale and $|r\rangle$ indicates the $r$ th system eigenstate. The environmental mode at frequency $\omega$ is annihilated (created) by the operator $a_{\omega}\left(a_{\omega}^{\dagger}\right)$. We also neglect a compensating term in (1) (see Ref. [24]). The system-environment coupling is given by

$\varphi_{n r}= \begin{cases}0.1 \mathrm{e}^{-|n-r| / R} & \text { if } n+r=\text { odd } \\ 0 & \text { if } n+r=\text { even. }\end{cases}$

$R=10$ is the range of dipole coupling. The frequency dependent part of the system-environment coupling is separately denoted by $\eta_{\omega}$. The energies are dimensionless in the same absolute energy scale as $E_{n}$ 's. The environment is characterised by a thermal spectral function $I(\omega)=$ $\eta_{\omega}^{2}\left(2 n_{\omega}+1\right)$ where $n_{\omega}$ is the bosonic distribution. Since our results are confined to zero temperature and the environment is in thermal equilibrium, the bare distribution $n_{\omega}=0$, and therefore $I(\omega)=\eta_{\omega}^{2}$ for which we use a Lorentzian spectral model below.

\subsection{The master equation}

The system is initially prepared in the qubit subspace $|\psi(0)\rangle=a|1\rangle+b|2\rangle \quad$ where $a=\sqrt{0.1}, b=\mathrm{e}^{\mathrm{i} \pi / 2} \sqrt{0.9}$. The system and the environmental degrees of freedom are initially decoupled. The master equation is solved for the MLS interacting with a zero temperature environment in the Born-Oppenheimer approximation [25,26] (BOA) $\hat{\tilde{\rho}}(t)=\hat{\tilde{\rho}}^{(S)}(t) \otimes \hat{\tilde{\rho}}_{e}(0)$. Here $\sim$ denotes the interaction picture, $\tilde{\rho}^{(S)}(t)$ denotes the reduced density matrix (RDM) of the system at time $t$ and $\rho_{e}$ is the density matrix of the pure environment. In the short time regime BOA is nearly manifest. For the approximation to hold at long times, the equilibration time of the environment should be longer than the exponential decoherence rates. The RDM is found as usual by the partial trace over the environment as

$\frac{\mathrm{d}}{\mathrm{d} t}\left(\tilde{\rho}^{(S)}\right)_{n m}(t)=-\int_{0}^{t} \mathrm{~d} t^{\prime} \sum_{r s} K_{r s}^{n m}\left(t, t^{\prime}\right)\left(\tilde{\rho}^{(S)}\right)_{r s}\left(t^{\prime}\right)$,

where

$$
\begin{aligned}
& K_{r s}^{n m}(\left.t, t^{\prime}\right) \\
&=\left\{F\left(t-t^{\prime}\right)\left[\left(\tilde{\varphi}(t) \tilde{\varphi}\left(t^{\prime}\right)\right)_{n r} \delta_{s, m}-\left(\tilde{\varphi}\left(t^{\prime}\right)\right)_{n r}(\tilde{\varphi}(t))_{s m}\right]\right. \\
&\left.\quad+F^{*}\left(t-t^{\prime}\right)\left[\left(\tilde{\varphi}\left(t^{\prime}\right) \tilde{\varphi}(t)\right)_{s m} \delta_{r, n}-(\tilde{\varphi}(t))_{n r}\left(\tilde{\varphi} t^{\prime}\right)_{s m}\right]\right\}
\end{aligned}
$$

is the non-Markovian system-noise kernel. For a 2LA it is easy to verify that $K_{r s}^{n m}\left(t, t^{\prime}\right)=K_{r s}^{n m}\left(t-t^{\prime}, 0\right)$. In a MLS the time translational symmetry is lost by the presence of a variety of possible transitions. Here $(\tilde{\varphi}(t))_{n r}=$ $(\tilde{\varphi}(0))_{n r} \mathrm{e}^{-i\left(E_{n}-E_{r}\right) t}$.

\subsection{The environmental correlator $F(t)$}

The environmental spectrum is introduced via the noise correlator as,

$F(\tau)=\Theta(\tau) \int_{-\infty}^{\infty} \mathrm{d} \omega \mathrm{e}^{\mathrm{i} \omega \tau}\left[\frac{A}{\pi} \frac{\varepsilon}{\left(\omega-\omega_{0}\right)^{2}+\varepsilon^{2}}\right]$,

where $\Theta$ is the Heaviside step function and the term in brackets is $I(\omega)$; viz. the environmental spectrum for which we adopt a Lorentzian form. The physical parameters of the Lorentzian spectrum are then the central frequency $\omega_{0}$ 
and the spectral width $\varepsilon$ of which the effects on the decoherence time scales can be independently examined. The spectral area is denoted by $A=\int_{-\infty}^{\infty} \mathrm{d} \omega I(\omega)$ which is nothing but a multiplicative factor in the system environment coupling in Eq. (4). The special choice of the Lorentzian is made for that, the three parameters, the area $A$, the central frequency $\omega_{0}$ and the width $\varepsilon$ can all be studied independent from each other in compliance with the purpose of this work. The model spectrum in Eq. (5) is unrealistic as a physical noise unlike the power exponential or the power Gaussian [10] which are more conventionally used for modelling the physical spectra. The conclusion driven from the spectrum (5) will however be general and applicable to the different physical models of environmental noise.

\section{The short-time decoherence}

It is easy to show that the decohering density matrix starts with a time evolution which is Gaussian-like given by

$\tilde{\rho}_{n m}^{(S)}(t) \simeq \sum_{r s}\left[\delta_{n r} \delta_{s m}-\frac{t^{2}}{2} K_{r s}^{n m}(0,0)\right] \tilde{\rho}_{r s}^{(S)}(0)$

where the Gaussian decoherence rate enters as a sum over the square roots of the positive eigenvalues of a characteristic operator $\hat{K}(0,0)$ representing the system-noise kernel. In Ref. [24] the positive definiteness of $\hat{K}(0,0)$ and its relation to $K_{r s}^{n m}(0,0)$ is demonstrated. This indicates the existence of a Gaussian-like decoherence time scale at short times. $K_{r s}^{n m}(0,0)$ in the right hand side of (6) is, by Eq. (4) proportional to the squared dipole coupling constants and $F(0)$. The latter is, by Eq. (5), the total area under the

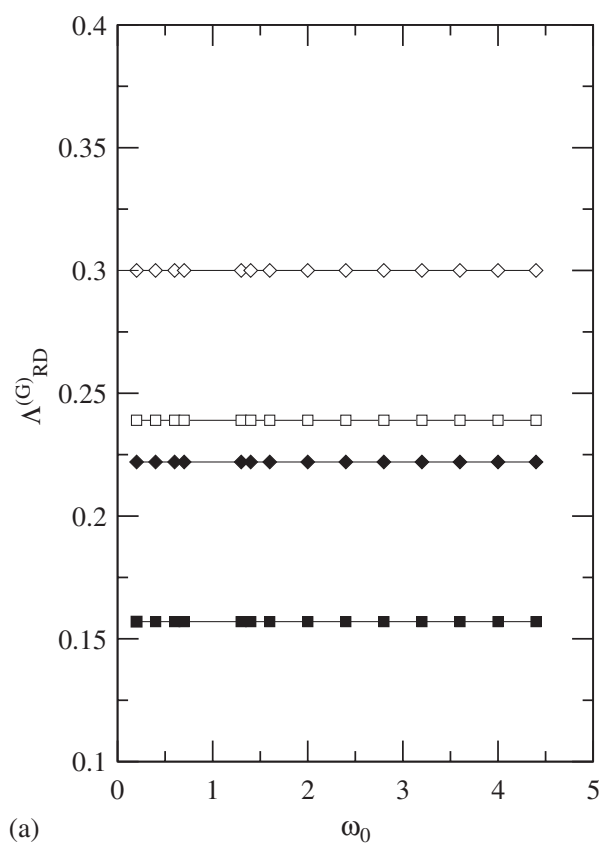

spectral function. Hence, the short time decoherence rate is expected to have contributions not only from the resonant terms but from the entire spectrum as a whole. We thus expect that for all components of the RDM Gaussian decoherence rates $\propto$ (spectral area $)^{1 / 2}$. The Gaussian decoherence rates $\Lambda_{\mathrm{RDL}}^{(G)}$ corresponding to the relaxation, dephasing and leakage contributions differ only in their dependence on the sum over the allowed dipole couplings $(\varphi)_{n r}$. Additionally, there is only a dependence on the spectral area with the other spectral parameters, i.e. spectral center $\omega_{0}$ and the width $\varepsilon$ being ineffective in short times (see Figs. 1-3).

The Gaussian decoherence times are easily calculated within a short time interval which corresponds in this work to $0 \leqslant t \leqslant 0.1$ [in units of the inverse absolute energy scale defined in Eq. (1)]. The numerically found RDL rates are shown for the model system in Eq. (1) in Fig. 1(a) for their dependence on the position, and Fig. 1(b) for their dependence on the shape of the spectrum for three and five level systems. Fig. 1(a,b) provide identical decay rates independently of the central frequency $\omega_{0}$ and the shape parameter $\varepsilon$ for fixed spectral area. These results are already conclusive for the dominance of the off-resonant processes at short times. Before examining the dependence on the spectral area, we examine the effect of the higher levels in the system as shown in Fig. 2. All levels are coupled by the dipole matrix in Eq. (2) with $R=10$. Increasing number of levels increase the decoherence times for $N \leqslant R$ followed by a saturation for $R<N$ which is due to the negligible contribution of the couplings out of the range.

The spectral area $A$ strongly affects the short time decoherence. Finally, in Fig. 3, we observe that all RDL

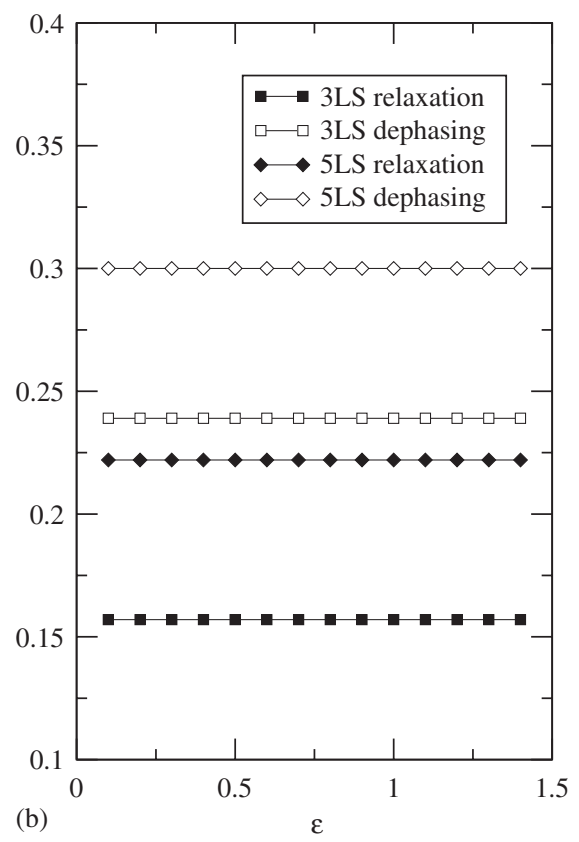

Fig. 1. Gaussian RDL rates with respect to (a) central frequency $\omega_{0}$ (with $A=1$ and $\varepsilon=0.1$ ), and (b) spectral width $\varepsilon$ (with $A=1$ and $\omega_{0}=2.4$ ). Note that the results depicted in this figure are independent of the shape of the spectrum. 
rates have power law dependence on the spectral area (a $\log -\log$ plot indicates that $\Lambda_{\mathrm{RDL}}^{(G)} \sim \sqrt{A}$ ) verifying once more the dominating role of the off-resonant transitions between the levels. From Figs. 1-3, it is necessary to conclude that, the short time decoherence rates are unconventionally non exponential characterized by the short time non-Markovian correlations in the spectrum.

\section{Leakage effects}

There are recent experiments where anomalies have been reported in the Rabi oscillation dynamics indicating a clear breakdown of the two-level approximation and strong short

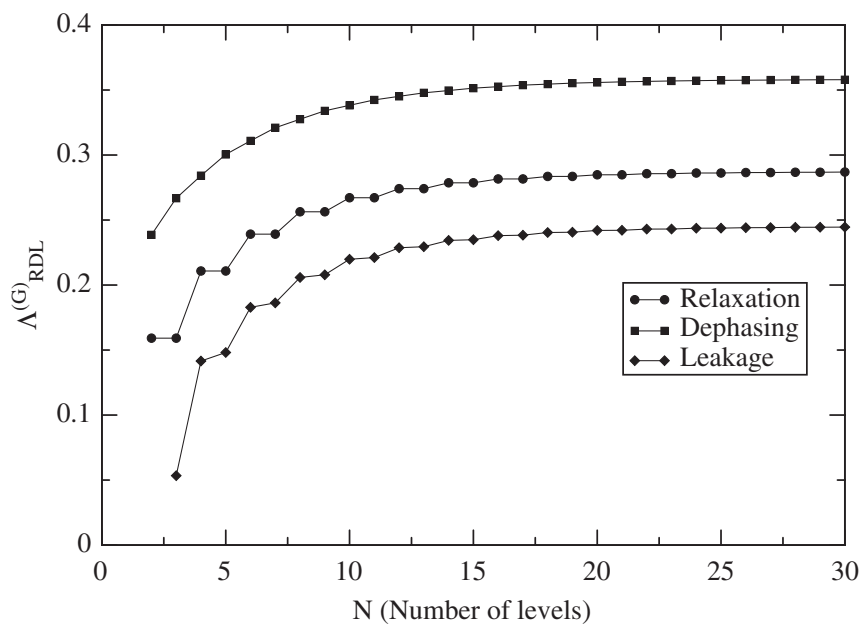

Fig. 2. Gaussian RD rates as a function of the number of system levels N. The coupling range is taken to be $R=10$. The stepwise behaviour in the rates for the first few levels is due to the forbidden (allowed) transitions by the matrix elements in Eq. (2). Spectral parameters are $A=1, \omega_{0}=1.0$ and $\varepsilon=0.1$ time leakage effects. In the first experiment by Zrenner et al. [27], single self-assembled excitonic q-dots are used to create excitons by a strong Rabi field which are then tunelled out and transformed into a photocurrent. The anomaly appears in the damping of the Rabi induced oscillations in the pulse averaged photocurrent as the area under the Rabi pulse is increased. This effect within the fixed time of the short $1 \mathrm{ps}$ pulse (dephasing and relaxation times are reported to be approximately $500 \mathrm{ps}$ ) could not be observed in a purely two levelled system as it would violate the fundamental principle of unitarity. The pulse width is shorter than the decoherence time scale by three orders of magnitude and it is clear that the observed damping does not originate from decoherence. The short time scale of the effect indicates the influence of the higher excitonic states. In a simple approach it has been shown that the damping of the oscillations as the intensity of the short pulse is increased, is due to the off-resonant leakage into biexcitonic levels [28,29]. Taking Ref. [28] as the basis of a physical model for this experiment, we show that the results are the manifestations of short-time nonresonant processes.

We add a Rabi coupling term in Eq. (1)

$H_{\mathrm{R}}=\sum_{n r=1}^{N} \varphi_{n r}|n\rangle\langle r|\left(\alpha(t) \mathrm{e}^{-i \Omega_{\mathrm{R}} t}+\right.$ h.c. $)$,

where $\alpha(t)$ and $\Omega_{\mathrm{R}}$ are the complex pulse amplitude and the frequency of the coherent Rabi field, respectively. We use rectangular and real pulse $\alpha(t)=\alpha \Theta\left(t_{\mathrm{P}}-t\right)$ where $t_{\mathrm{P}}=1$ in units of the fundamental energy scale. This corresponds to $t_{\mathrm{P}} \simeq 1 \mathrm{ps}$ when $E_{2}-E_{1} \simeq 1 \mathrm{meV}$ in Zrenner et al.'s experiment. We solve the master equation for $N=3$ and neglect the environment. Then the time dependence becomes a function of the total pulse area $\alpha$. The result
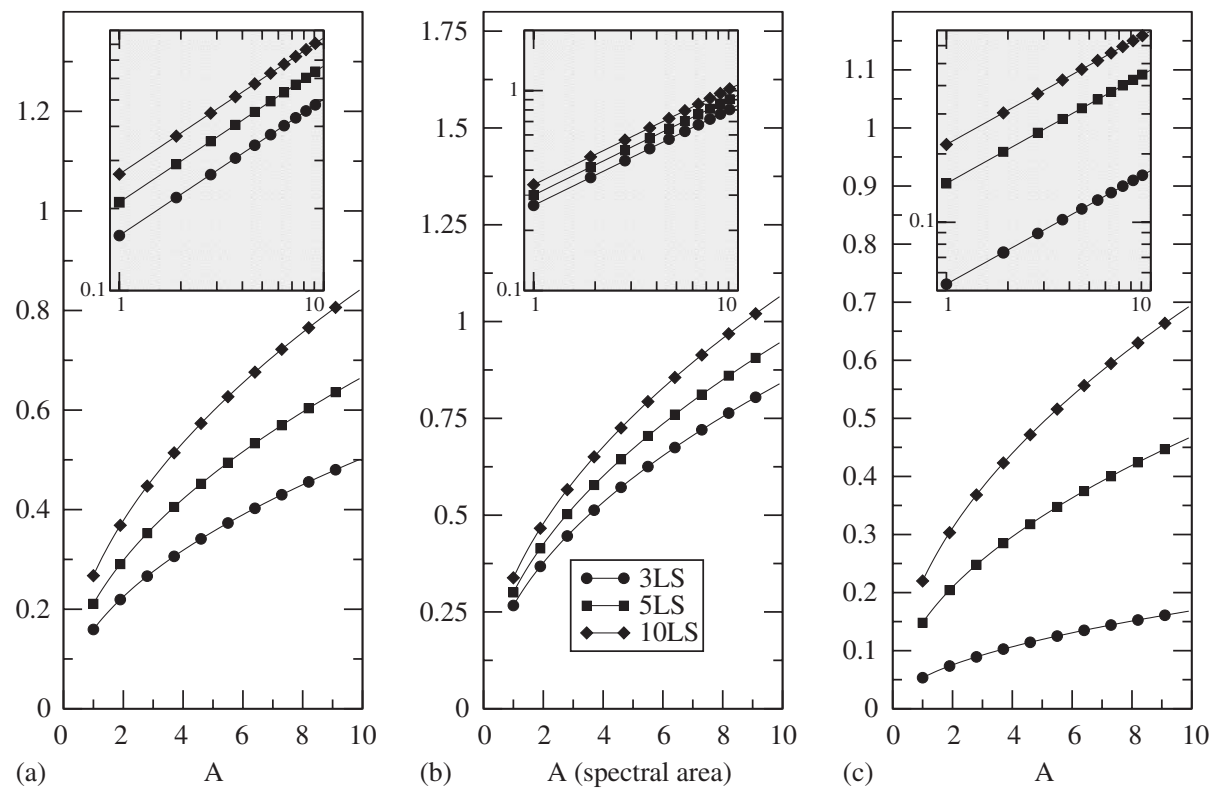

Fig. 3. Dependence of the short time Gaussian rates for (a) R, (b) D, (c) L on the spectral area. On a log-log scale, as shown in the inlets, the dependence is $\sim \sqrt{A}$. 


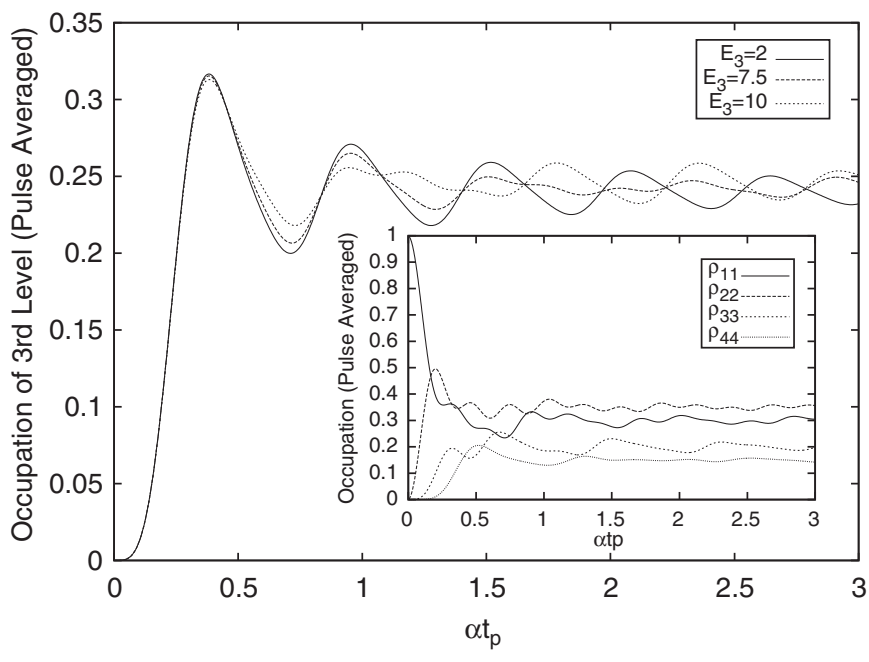

Fig. 4. The time average over the Rabi pulse of the third level occupancy is shown as a function of $\alpha t_{\mathrm{P}}$. Three curves refer to three different third level resonant energy $E_{3}=10,7.5,2$ with $E_{2}=2$ and $E_{1}=1$. The Rabi field is in resonance with the first two levels. In the inlet the four level occupancies are shown for equally spaced levels with a Rabi field resonantly coupling the neighbouring levels.

is then averaged over the pulse as in the experiment. In Fig. 4 the pulse-averaged occupation of this non-resonantly coupled third level, [i.e. $\left.\left\langle\rho_{33}(t)\right\rangle_{\text {pulse }}=1 / t_{\mathrm{P}} \int_{0}^{t_{\mathrm{P}}} \mathrm{d} t \rho_{33}(t)\right]$ is shown as a function of the pulse area when the Rabi field is resonant with the first two levels for three different third level energies. In the figure, all three curves indicate pulse averaged third level occupation, i.e. $\left\langle\rho_{33}(t)\right\rangle_{\text {pulse }}$, for the third level energies $E_{3}=2,7.5,10$. In the first one, $E_{3}$ is degenerate with $E_{2}=2$, and, for the last one, $E_{3}=10$ is supposedly close to an ideal two level system $\left(E_{2}-E_{1}\right) \ll$ $\left(E_{3}-E_{2}\right)$. The occupation of the third level is clearly observed to be largely unaffected by the large range of the energies for the third level. Another point is that, the peak position occurs at $t_{\mathrm{P}} \leqslant 1 / \alpha$. Using (2), this implies $t_{\mathrm{P}} \leqslant \varphi_{12} T_{\mathrm{R}} /(2 \pi)$, where $T_{\mathrm{R}}$ is the Rabi oscillation period, therefore $t_{\mathrm{P}} \simeq 0.1 T_{\mathrm{R}}$ concluding that the third level is already occupied maximally before the completion of a single Rabi period. This short time effect is counterintuitive from the traditional way of thinking in terms of the longtime resonant transitions. It must be remarked that this is an exact result. It appears that a multilevelled system decides to act like so at very short times in comparison with typical resonant time scales. Thus Fig. 4, in confirmation of the earlier theoretical calculations [28], manifests the effect of the strong influence of the nonresonant processes on leakage. A second experiment supporting strong nonresonant leakage at short times has been recently made on semiconductor NMR devices by Yusa et al. [30] where offresonant multiple quantum coherence effects between levels separated by more than one quantum of nuclear spin quantum number were observed. This experiment is the four level version of the quantum dot experiment by Zrenner et al. done with stable nuclei with total spin $\frac{3}{2}$ in which the short time multilevel dynamics can be probed.
The results are shown in the inlet of Fig. 4. The first two levels are already nearly fully occupied for $t_{\mathrm{P}} \simeq 0.2 / \alpha$ when the third level is $10 \%$ full and the fourth level is empty. Within another short time, i.e. at $t_{\mathrm{P}} \simeq 0.5 / \alpha$, the third and the fourth levels are already saturated at about $20 \%$.

The results in this section are, in support of the previous sections in that, the multistate dynamics cannot be ignored at short time scales. The reconfiguration of the level occupancies occurs at short times and is comparable to the decoherence time scales.

\section{Conclusions}

It is shown that decoherence is dominated by the short time off resonant processes. Such processes are induced by the entire frequency range in the noise spectrum. It is demonstrated that off resonant processes also cause a strong short time leakage in multilevelled systems and this very reason disvalidates the applicability of the two-level approximation in most realistic systems.

\section{Acknowledgements}

This research is supported by the Scientific and the Technical Research Council of Turkey (TÜBITAK) grant number TBAG-2111 (101T136).

\section{References}

[1] A.J. Leggett, S. Chakravarty, A.T. Dorsey, M.P.A. Fisher, A. Garg, W. Zwerger, Rev. Mod. Phys. 59 (1987) 1.

[2] D. Loss, D.P. DiVincenzo, Exact Born Approximation for the spinboson model (cond-mat/0304118).

[3] T. Vorrath, T. Brandes, B. Kramer, Dynamics of a large spin-boson system in the strong coupling regime (cond-mat/0111220).

[4] C. Anastopoulos, B.L. Hu, Phys. Rev. A 62 (2000) 033821.

[5] M. Dube, P.C.E. Stamp, Chem. Phys. (2001) also (cond-mat/ 0102156) (Quantum Physics of Open Systems, Special Issue)

[6] P.C.E. Stamp, I.S. Tupitsyn, Chem. Phys. 296 (2004) 281.

[7] A.O. Caldeira, A.J. Leggett, Phys. Rev. Lett. 46 (1981) 211.

[8] A.O. Caldeira, A.J. Leggett, Ann. Phys. 149 (1983) 374.

[9] A.J. Leggett, A. Garg, Phys. Rev. Lett. 54 (1985) 857.

[10] T. Hakioğlu, K. Savran, Phys. Rev. B 71 (2005) 115115.

[11] G. Burkard, R.H. Koch, D.P. Di Vincenzo, Phys. Rev. B 69 (2004) 064503.

[12] F. Bloch, Phys. Rev. 105 (1957) 1206.

[13] A.G. Redfield, IBM J. Res. Dev. 1 (1957) 19.

[14] G. Lindblad, Commun. Math. Phys. 48 (1976) 119.

[15] D.P. DiVincenzo, D. Loss, Phys. Rev. B 71 (2005) 035318.

[16] R.V. Voss, R.A. Webb, Phys. Rev. Lett. 47 (1981) 265.

[17] J.M. Martinis, M.H. Devoret, J. Clarke, Phys. Rev. Lett. 89 (1985) 207601.

[18] J.R. Friedman, V. Patel, W. Chen, J.E. Lukens, Nature (London) 406 (2000) 43.

[19] D. Natelson, R.L. Willett, K.W. West, L.N. Pfeiffer, Phys. Rev. Lett. 86 (2001) 1821

[20] P. Cedraschi, V.V. Ponomarenko, M. Büttiker, Phys. Rev. Lett. 84 (2000) 346.

[21] J.J. Lin, N. Giordano, Phys. Rev. B 35 (1987) 1071.

[22] P. Mohanty, E.M.Q. Jariwala, R.A. Webb, Phys. Rev. Lett. 78 (1997) 3366.

[23] P. Mohanty, R.A. Webb, Phys. Rev. B 55 (1997) R13452. 
[24] K. Savran, T. Hakioğlu, H. Sevinçli, E. Meșe, J. Phys. Condens. Mat. (2005), to appear.

[25] M. Born, J.R. Oppenheimer, Ann. Phys. 84 (1927) 457.

[26] A. Bohm, M. Loewe, Quantum Mechanics, Springer, Berlin, 2001.

[27] A. Zrenner, E. Beham, S. Stufler, F. Findeis, M. Bichler, G. Abstreiter, Nature 418 (2002) 612.
[28] J.M. Villas-Boas, S.E. Ulloa, A.O. Govorov, Phys. Rev. Lett. 94 (2005) 057404

[29] J.M. Villas-Boas, A.O. Govorov, S.E. Ulloa, Phys. Rev. B 69 (2004) 125342.

[30] G. Yusa, K. Muraki, K. Takashina, K. Hashimoto, Y. Hirayama, Nature 434 (2005) 1001. 